\title{
Anti-plasmodial Activity of a Non-protein Amino Acid Taurine
}

\author{
Thavamani Rajapandi*, Kazim Ackie, Kavita Rajeev Hegde \\ Department of Natural Sciences, Coppin State University, Baltimore MD, The United States \\ Email address: \\ trajapandi@coppin.edu (T. Rajapandi), kazimackie@gmail.com (K. Ackie), khegde@coppin.edu (K. R. Hegde) \\ ${ }^{*}$ Corresponding author
}

\section{To cite this article:}

Thavamani Rajapandi, Kazim Ackie, Kavita Rajeev Hegde. Anti-plasmodial Activity of a Non-protein Amino Acid Taurine. Biomedical Sciences. Vol. 5, No. 3, 2019, pp. 34-37. doi: 10.11648/j.bs.20190503.13

Received: September 26, 2019; Accepted: October 15, 2019; Published: October 25, 2019

\begin{abstract}
Human malaria is caused by a few selected species of the genus Plasmodium. Among these, Plasmodium falciparum causes almost $90 \%$ of malaria-related mortality. Novel anti-malarial compounds are hence required to fight the anti-malarial drug-resistant $P$. falciparum parasites. The objective of this study is to analyze the effectiveness of Taurine (2aminoethane sulfonic acid), a non-protein amino acid, in preventing the growth and development of both asexual and sexual stages of in vitro cultured $P$. falciparum parasites. We found that $200 \mathrm{mM}$ concentration of Taurine almost completely $(>80 \%)$ inhibited the propagation of asexual stages of $P$. falciparum. In contrast, it did not have any inhibitory activity against the maturation of sexual or gametocyte stages. However, the gametocytogenesis or the conversion of asexual to stage I gametocyte was blocked partially by this compound. The results suggest that derivatives of Taurine /2-aminoethane sulfonic acid could be considered to further improve the effectiveness of Taurine as an antimalarial compound against both the asexual and early sexual stages of $P$. falciparum.
\end{abstract}

Keywords: Malaria, Plasmodium falciparum, 2-amino Ethane Sulfonic Acid (Taurine), Drug Resistance and Gametocytes

\section{Introduction}

Protozoan parasites cause several pathological manifestations in humans, malaria being one of the most prevalent. Among the five species of Plasmodium parasites that cause malaria in humans, Plasmodium falciparum is the deadliest while $\mathrm{P}$. vivax is the most widespread [1]. Numerous strategies have been developed and applied to control the spread of malaria parasites. Approaches such as spraying of insecticides both indoors and outdoors, use of insecticide treated bed nets (ITNs), and antimalarial drugs for prevention as well as treatment have long been used to control the spread of both Plasmodium parasites and the mosquito vectors which transmit malaria among humans. A greater success has been achieved by these approaches to control malaria but none of these approaches have effectively blocked the transmission of malaria parasites and prevented the spread of malaria. Also, P. falciparum parasites have acquired resistance to almost all anti-malarial drugs used so far [1-6]. Currently, the first line of treatment for most of the $P$. falciparum malaria is artemisinin-based combination therapy (ACT). But very recently, P. falciparum acquired resistance to artemisinin $[1,3,7-10]$, and still the combination therapy is the only viable option available to treat malaria [1]. Development of anti-malarial vaccine against various targets and attenuated whole parasites is underway in several laboratories, but the preliminary clinical efficacy data are not very promising for an effective and complete control and elimination of malaria. Therefore, additional approaches and novel anti-malarial drugs are warranted to combat this deadly disease.

In this study, we analyzed whether certain nutraceuticals can be used to control the spread of $P$. falciparum malaria. For several decades, studies have been done on nutrition and nutritional supplements towards the control of several pathogens which inflict illness in human populations [11]. Several supplements have been found to effectively kill protozoan parasites which cause various diseases in humans, such as trypanosomiasis, which is caused by, Trypanosoma brucei Interestingly, Taurine, 2-amino ethane sulfonic acid, was tested against rodent malaria parasite $P$. chabaudi. It was found that high concentration of Taurine eliminates $P$. 
chabaudi parasites in rodents [12]. Delic et al. [12] also found that mice defective in taurine transport (taut ${ }^{-1)}$ have lost their ability to fight against blood-stage infections with Plasmodium chabaudi malaria, and $90 \%$ of the control taut ${ }^{+/+}$mice survive [12]. But the effect of taurine against $P$. falciparum growth was never tested previously. In this study, we tested the effectiveness of Taurine, a nutraceutical, against the asexual and sexual stages of growth and development of $P$. falciparum parasites. We found that Taurine blocked asexual growth and development of $P$. falciparum almost completely but did not affect sexual stages.

\section{Materials and Methods}

\subsection{Materials}

Taurine (Cat \# T8691) was purchased from Sigma Aldrich (Thermo Fisher), RPMI medium supplemented with Hypoxanthine and glucose was gifted by K. D. Medicals (KD MEDICALS, Columbia MD, USA (Cat \# CUS-0645)), Type A Human serum and type $\mathrm{O}^{+}$erythrocytes were obtained from Interstate Blood Bank (Interstate Blood Bank, Inc. Memphis, TN Center). Mixed gas containing 3\% $\mathrm{O}_{2}, 5 \% \mathrm{CO}_{2}$ and $92 \%$ Nitrogen was purchased from Roberts Oxygen (Roberts Oxygen, Baltimore MD), Giemsa stain (cat \# GS1L) was purchased from Sigma Aldrich. The concentrated Giemsa stain was diluted to $5 \%$ in $\mathrm{H}_{2} \mathrm{O}$ at the time of use.

\subsection{Methods}

\subsubsection{Preparation of Complete Culture Medium}

Four hundred and fifty $\mathrm{ml}$ RPMI medium was mixed with $45 \mathrm{ml}$ of serum, $16 \mathrm{ml}$ of $7.5 \%$ sodium bicarbonate and $0.01 \%$ Gentamicin. The culture medium was filtered through 0.22 $\mu \mathrm{M}$ filter unit (Thermo Scientific, Cat \# 5660020) and stored at $4{ }^{\circ} \mathrm{C}$ for $2-3$ weeks. Culture medium containing various concentrations of Taurine was prepared separately, by dissolving the required amount of Taurine in complete culture medium, filtered through $0.22 \mu \mathrm{M}$ filter unit, when needed.

\subsubsection{In Vitro Culture of P. falciparum}

$P$. falciparum NF54 isolate was obtained from Johns Hopkins Malaria Research Institute. P. falciparum NF54 was cultured in RPMI medium supplemented with $8 \% \mathrm{O}^{+}$human serum (heat inactivated) and Sodium bicarbonate. $P$. falciparum NF54 culture was maintained in RPMI by daily media change. The growth of NF54 was monitored by daily blood smearing, Giemsa staining of methanol fixed slides, and microscopy.

\subsubsection{In Vitro Culture of P. falciparum Gametocytes}

$P$. falciparum NF54 gametocytes were cultured as described previously [13]. The development and maturation of various stages of gametocytes were monitored by daily blood smearing, Giemsa staining of methanol fixed smears, and microscopy.

\subsubsection{Analysis of the Maturation of Gametocytes}

$P$. falciparum NF54 gametocyte culture was initiated in $6 \%$ hematocrit (HCT) and $0.2 \%$ of a sorbitol synchronized asexual ring stage parasite in complete culture medium. After 24 hours, the culture was diluted to $3 \% \mathrm{HCT}$ and aliquoted into 6 flasks containing $20 \mathrm{ml}$ of complete CM. Taurine treatment of these cultures were followed from Day 2 to 16 as described in Table 1 .

\section{Results}

\subsection{Effect of Taurine on P. falciparum Development}

The growth of $P$. falciparum NF54 parasite was initiated from an established culture and tested the effect of Taurine on the growth and development of $P$. falciparum NF54. Actively growing $P$. falciparum NF54 culture was diluted to $0.2 \%$ parasitemia with $4 \% \mathrm{HCT}$ in six $25 \mathrm{~mm}$ culture flasks containing $20 \mathrm{ml}$ culture medium. Various concentrations, 1 , 2.5, 5.0, 10 and $20 \mathrm{mM}$ of Taurine (diluted from a stock of $500 \mathrm{mM}$ Taurine in $\mathrm{CM}$ ) were added and exchanged with $3 \%$ $\mathrm{O}_{2}$ containing mixed gas. One flask containing $20 \mathrm{ml}$ of $\mathrm{CM}$ with the same parasite and HCT without any added Taurine was set up as positive control. CM containing various concentrations of Taurine was exchanged every 24 hours for 4 days. The growth was monitored everyday by Giemsastained thin blood smears. Our results indicate that even with $20 \mathrm{mM}$ concentrations of Taurine, there was no significant inhibition or acceleration of the growth of NF54 and the parasitemia was almost similar in all cultures (data not shown).

Since the first set of experiments, up to $20 \mathrm{mM}$ concentration of Taurine, did not show any significant effect on the asexual growth of $P$. falciparum, we tested the effect of higher concentrations of Taurine on the growth and development of asexual stages. For this experiment, 25, 50, 100, 200- and 500-mM concentrations of Taurine were prepared in CM (Taurine was dissolved directly in culture medium, filtered through $0.22 \mu \mathrm{M}$ filtration unit), and the cultures were initiated in $20 \mathrm{ml}$ with $0.2 \% P$. falciparum NF54. These cultures were maintained for 96 hours and the parasitemia was calculated from Giemsa-stained thin blood smears. Blood smears were blinded and the parasitemia was calculated by two independent investigators. As shown in figure 1, higher concentrations such as 100- and 200-mM taurine blocked more than $80 \%$ of the parasite growth in 96 hours. At the concentrations of 500-mM Taurine, the growth of NF54 was completely blocked. Additional experiments by using a few $P$. falciparum lines will be required to determine the IC50 of Taurine against the asexual growth and development of $P$. falciparum. 


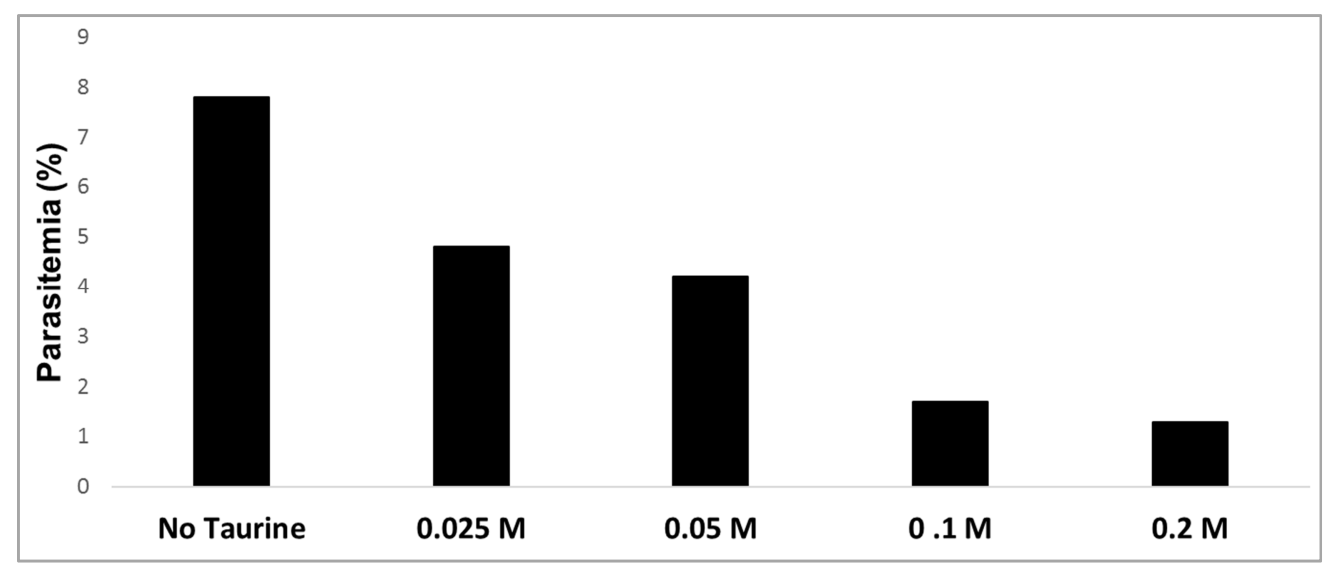

Figure 1. Effect of Taurine on asexual stages of P. falciparum growth.

P. falciparum parasite isolate NF54 was grown in complete culture medium containing $0.025,0.05,0.1$ and $0.2 \mathrm{M}$ concentrations of Taurine for 96 hours. Thin blood smears were prepared, fixed in methanol, stained with Giemsa and the parasitemia was calculated by counting $P$. falciparum infected red blood cells in 1000 red blood cells per experimental slide to determine the Parasitemia (number of parasite infected RBC in 100 RBCs.

\subsection{Effect of Taurine on P. falciparum Sexual Stages}

Gametocytes are the transmissible forms of malaria parasites to mosquito vectors from vertebrate hosts and are developed in red blood cells $[14,15]$. All the available antimalarial drugs, except primaquine, do not have an effect on gametocytes. To test the gametocidal activity of Taurine, gametocyte cultures were initiated as described previously in $6 \%$ HCT (stress induction by high HCT). After 24 hours, the gametocyte culture was diluted to $2.5 \%$ HCT and divided into 6 portions. We tested $200 \mathrm{mM}$ concentrations of Taurine on various developmental stages of gametocyte as detailed in Table 1. The culture medium containing Taurine was exchanged every 24 hours for 15 days. The gametocyte development was monitored by blood smearing and microscopy. As shown in Table 1, $200 \mathrm{mM}$ concentrations of taurine did not block the maturation of most stages of gametocytes and all Taurine treated cultures showed similar number of gametocytes except the one culture where asexual stages are treated with $200 \mathrm{mM}$ concentrations of Taurine.

Table 1. Effect of Taurine on the maturation of sexual stages of P. falciparum.

\begin{tabular}{llllllll}
\hline Day-0 & Day-2 & Days-3\&4 & Days-5\&6 & Days-7\&8 & Days-9\&10 & Days-11-15 & Gametocytemia (\%) on Day 16 \\
\hline Fl-1 & CM & CM & CM & CM & CM & CM \\
FL-2 & CM & CM & CM & CM & CM-T & CM-T \\
FL-3 & CM & CM & CM & CM-T & CM-T & CM-T \\
FL-4 & CM & CM & CM-T & CM-T & CM-T & CM-T \\
FL-5 & CM & CM-T & CM-T & CM-T & CM-T & CM-T \\
FL-6 & CM-T & CM-T & CM-T & CM-T & CM-T & CM-T & 1.9 \\
\hline
\end{tabular}

P. falciparum NF54 was cultured to $6 \%$ of asexual ring stage and synchronized with D-sorbitol to lyse asexual tophozoites and schizonts. The synchronized ring-stage parasite culture was diluted to $3 \%$ and aliquoted into 6 flasks (FL) containing $20 \mathrm{ml}$ of culture medium (CM), and marked as FL1, FL2, FL3, FL4, FL5 and FL6. Gametocyte maturation in these cultures were tested against 0.2 M Taurine as follows. FL-6 was set up to monitor the inhibitory effect on StageI, FL-5 was set up to monitor the inhibitory effect on Stage- I to II, FL-4 was set up to monitor the inhibitory effect on Stage -II to III, FL- 3 was set up to monitor the inhibitory effect on Stage III to IV, FL-2 was set up to monitor the inhibitory effect on Stage IV to V, FL-1 was set up to monitor the normal growth and development of PfNF54 without any added Taurine.

\section{Discussion}

P. falciparum developmental stage-specific growth inhibition by Taurine suggests Taurine interaction may be target specific on the asexual stage parasites. Importantly, Lutgen P [11] finds that, in mice, when the circulating Taurine concentration falls below the physiological level, Plasmodium chabaudi infected mice develop a $60 \%$ higher parasitemia [11]. Also, Taurine accumulation increased in Plasmodium infected RBCs compared with uninfected RBCs [11, 16], and this result suggests that Taurine accumulation in human RBCs may use nutrient permeability pathways (NPP) and EXP1 [16-18] for the transport of Taurine and this transport system may not support Taurine uptake in gametocytes.

\section{Conclusion}

The present study results demonstrate that there is a positive correlation between Taurine concentration and its inhibitory effect on the growth and development of asexual stages of $P$. falciparum parasite. Interestingly, Taurine did not show inhibitory activity on the maturation of $P$. falciparum sexual forms or gametocytes.

\section{Recommendations}

The findings of this study suggest that $P$. falciparum growth inhibition analysis by other compounds like Taurine 
or modified derivatives of Taurine may provide valuable information on the mechanism of growth inhibition of $P$. falciparum parasites by Taurine and its derivatives. The evaluation of various other food supplements which contain Taurine on the growth inhibition of Plasmodium parasites may also be informative to design and develop novel class of anti-malarial compounds.

\section{Acknowledgements}

Plasmodium falciparum NF54 was contributed by A. Tripathi at the JHMRI, Baltimore MD.

\section{Authors Contribution}

TR and KH conceived and devised the study, designed experiments. AK and TR performed experiments, collected data and analyzed results. TR and $\mathrm{KH}$ wrote the manuscript.

\section{Ethical Approval}

The research study was conducted as per the National Institutes of Health $(\mathrm{NIH})$ guidelines on using blood and blood borne pathogens. This study was conducted in a Bio Safety Level (BSL2) facility approved by the institution. Animals were not used in this study. Human subjects were not involved in this study.

\section{Conflict of Interest}

The author reports no conflict of interest.

\section{Consent for Participation}

Not applicable. Human subjects did not participate in nor were involved in this study. The blood and serum samples used in this study were obtained from Interstate Blood Bank (Interstate Blood Bank, Inc. Memphis, TN Center).

\section{References}

[1] Organization WHO. 2016. World malaria report 2016.

[2] Bousema JT, Schneider P, Gouagna LC, Drakeley CJ, Tostmann A, Houben R, Githure JI, Ord R, Sutherland CJ, Omar SA, Sauerwein RW. 2006. Moderate effect of artemisinin-based combination therapy on transmission of Plasmodium falciparum. J Infect Dis. 193: 1151-1159.

[3] Mockenhaupt FP, Ehrhardt S, Dzisi SY et al. 2005. A randomised, placebo-controlled, and double-blind trial on sulfadoxine-pyrimethamine alone or combined with artesunate or amodiaquine in uncomplicated malaria. Tropical Medicine and International Health 10, 512-520.

[4] Oesterholt MJ, Alifrangis M, Sutherland CJ, Omar SA, Sawa P, Howitt C Gouagna L Sauerwein RW, Bousema T. 2009. Submicroscopic gametocytes and the transmission of antifolate-resistance Plasmodium falciparum in Western Kenya. PLoS One.
[5] Abdul-Ghani R, Basco LK, Beier JC, Mahdy M. 2015. Inclusion of gametocyte parameters in anti-malarial drug efficacy studies: filling a neglected gap needed for malaria elimination, Malaria Journal, 14: 413.

[6] Straimer, J., et al. "Drug Resistance. 2015. K13-Propeller Mutations Confer Artemisinin Resistance in Plasmodium falciparum Clinical Isolates." Science 347 428-43.

[7] Cheng Q, Kyle DE, Gatton ML. 2012. Artemisinin resistance in Plasmodium falciparum: A process linked to dormancy? Int J Parasitol Drugs Drug Resist. 2: 249-255.

[8] Dondorp, A. M., Nosten, F., Yi, P., Das, D., Phyo, A. P., Tarning, J., Lwin, K. M., Ariey, F, Hanpithakpong, W., Lee, S J., Ringwald, P., Silamut, K., Imwong, M., Chotivanich, K., Lim, P., Herdman, T., An, S. S., Yeung, S., Singhasivanon, P., Day, N. P., Lindegardh, N., Socheat, D., White, N. J., 2009. Artemisinin resistance in Plasmodium falciparum malaria. $\mathrm{N}$. Engl. J. Med. 361, 455-467.

[9] Teuscher, F., Gatton, M. L., Chen, N., Peters, J., Kyle, D. E., Cheng, Q., 2010. Artemisinin-induced dormancy in Plasmodium falciparum: duration, recovery rates, and implications in treatment failure. J. Infect. Dis. 202, 1362-1368.

[10] Teuscher, F., Chen, N., Kyle, D. E., Gatton, M. L., Cheng, Q., 2012. Phenotypic changes in artemisinin resistant Plasmodium falciparum lines in vitro: evidence for decreased sensitivity to dormancy and growth inhibition. Antimicrob. Agents Chemother. 56, 428-431.

[11] Lutgen P (2017) New Insights into Malaria Prophylaxis. Pharm Pharmacol Int J 5 (6): 00141. DOI: 10.15406/ppij.2017.05.00141.

[12] Delic D, Ulrich Warskulat, Elena Borsch, Saad Al-Qahtani, Saleh Al-Quraishi, Dieter Ha"ussinger, and Frank Wunderlich. 2010. Loss of Ability To Self-Heal Malaria upon Taurine Transporter Deletion Infection and Immunity, Vol. 78, No. 4, $1642-1649$.

[13] Tanaka TQ, Guiguemde WA, Barnett DS, Maron MI, Min J, Connelly MC, Suryadevara PK, Guy RK, Williamson KC. 2015. Potent Plasmodium falciparum gametocytocidal activity of diaminonaphthoquinones, lead antimalarial chemotypes identified in an antimalarial compound screen. Antimicrob Agents Chemother 59: 1389-1397.

[14] Drakeley C, Sutherland C, Bousema JT, Sauerwein RW, Targett AT. 2006. The epidemiology of Plasmodium falciparum gametocytes: weapons of mass dispersion. Trends Parasitol. 22: 424-30.

[15] Eksi S, Morahan BJ, Haile Y, Furuya T, Jiang H, et al. 2012. Plasmodium falciparum Gametocyte Development 1 (Pfgdv1) and Gametocytogenesis Early Gene Identification and Commitment to Sexual Development. PLoS Pathog 8 (10).

[16] Kirk K, Tilley L, Ginsburg H (1999) Transport and trafficking in the malaria infected erythrocyte. Parasitol Today 15 (9): 355-357.

[17] Mesén-Ramírez P, Bergmann B, Tran TT, Garten M, Stäcker J, Naranjo-Prado I, et al. (2019) EXP1 is critical for nutrient uptake across the parasitophorous vacuole membrane of malaria parasites. PLoS Biol 17 (9): e3000473.

[18] Dickerman, B. K. et al. Identification of inhibitors that dually target the new permeability pathway and dihydroorotate dehydrogenase in the blood stage of Plasmodium falciparum. (2016) Sci. Rep. 6, 37502; doi: 10.1038/srep37502. 